\title{
Senescence of Rice Leaves VI. Comparative Study of the Metabolic Changes of Senescing Turgid and Water-Stressed Excised leaves
}

\author{
Ching Huei Kao \\ Department of Agronomy, National Taiwan University, Taipei, \\ Taiwan, Republic of China
}

\begin{abstract}
Some metabolic changes of senescing turgid and water-stressed excised rice leaves were compared under incubation in the dark. The decrease of the chlorophyll and protein level and the increase of the $a$-amino nitrogen were faster in the water-stressed leaves than in the turgid leaves during the first two or three days of incubation. However, the changes in levels of chlorophyll, protein and $a$-amino nitrogen were later retarded by water stress. The rate of decline in soluble sugar was more rapid in the turgid than in the water-stressed leaves. In turgid leaves, there was a slight but significant increase in the proline content in the first day of incubation; subsequently, proline accumulated relatively rapidly, yet at a slower rate than in the stressed leaves. The activity of acid inorganic pyrophosphatase increased, but that of alkaline inorganic pyrophosphatase decreased during the senescence of both turgid and water-stressed leaves. The enzyme activities are, therefore, due to two separate enzyme proteins. Water stress enhanced the increase of acid inorganic pyrophosphatase activity only during the first two days; it retarded the decrease of alkaline inorganic pyrophosphatase activity at later stage of incubation.

It is concluded that water stress does not accelerate all the metabolic changes associated with the senescence of excised leaves.

Key words: Inorganic pyrophosphatases - Leaf senescence - Proline (senescence) - Rice leaf - Water stress.
\end{abstract}

Brady et al. (1974) demonstrated that early events in the senescence of turgid and water stressed leaves are similar. Some reports have shown that water stress enhanced the processes associated with the senescence of intact plants as well as of detached organs (Dwivedi et al. 1979a, 1979b, Brady et al. 1974, Gates 1968, Livne and Vaadia 1968, Shah and Loomis 1965). In a detailed study, however, Dwivedi et al. (1979a) concluded that water stress did not accelerate all the metabolic changes associated with leaf senescence, though they showed that the changes in the levels of chlorophyll and protein as well as $a$-amino nitrogen were greater in leaves subjected to water stress than in similar leaves kept turgid. Recently, we found that mannitol-stressed intact rice leaves had higher chlorophyll content than turgid intact leaves after four days of dark incubation (Kao 1979).

The present investigation was undertaken to understand whether all the metabolic changes connected with the senescence of excised rice leaves are enhanced by water stress. 


\section{Materials and Methods}

Rice (Oryza sativa cv. Taichung Native 1) seedlings were cultured as previously described (Kao 1980b). The apical $3 \mathrm{~cm}$ of the third leaves of 8-day-old seedlings were used for experiments.

The method of Stewart (1971) for subjecting leaf segments to water stress was applied with some modifications. Groups of ten leaf segments were exposed to the vapor above a $0.5 \mathrm{M} \mathrm{NaCl}$ solution, by spreading the leaves on water-proof paper boats, which were in turn kept over 4-layer-cheese cloth pads soaked with $20 \mathrm{ml}$ $\mathrm{NaCl}$ solution in Petri dishes. Comparable leaf samples were kept turgid by floating them in $20 \mathrm{ml}$ of distilled water to serve as the control. All samples were kept in darkness at $27^{\circ} \mathrm{C}$.

Maximum leaf width of turgid leaves was measured by ruler. The width of water-stressed leaves was rapidly measured under a stereoscopic microscope.

Chlorophyll, $a$-amino nitrogen and protein were extracted and determined as described before (Kao 1980a, 1980b). Total sugar was thoroughly extracted with boiling $80 \%$ ethanol and estimated by the anthrone reagent method (Yoshida et al. 1972). Proline was extracted and its concentration determined following the method of Bates et al. (1973). Leaf segments were homogenized with $3 \%$ sulfosalicylic acid and centrifuged. The supernatant was treated with acetic acid and acid-ninhydrin, boiled for $1 \mathrm{hr}$, and extracted with toluene. Then, its absorbance at $520 \mathrm{~nm}$ was read.

Inorganic pyrophosphatases (EC 3.6.1.1) were extracted as described by Rauser (1971). Acid inorganic pyrophosphatase activity was determined in $5 \mathrm{ml}$ reaction mixtures containing $5 \mu \mathrm{mol} \mathrm{NaPP}$; $300 \mu \mathrm{mol}$ citrate buffer, $\mathrm{pH} 5.0$; and $1 \mathrm{ml}$ enzyme extract. Alkaline inorganic pyrophosphatase activity was determined in $5 \mathrm{ml}$ reaction mixtures containing $5 \mu \mathrm{mol} \mathrm{NaPP}, ; 170 \mu \mathrm{mol}$ Tris buffer, $\mathrm{pH} 8.6$; $100 \mu \mathrm{mol} \mathrm{MgCl}_{2}$ and $0.1 \mathrm{ml}$ enzyme extract. After incubation at $37^{\circ} \mathrm{C}$ for $10 \mathrm{~min}$, the reaction was stopped by adding $1 \mathrm{ml} 20 \%$ perchloric acid. After centrifugation, an aliquot of the clean supernatant was taken for inorganic phosphorus determination (Yoshida et al. 1972). Pyrophosphatase activity was calculated on the basis of the net inorganic phosphorus released during incubation. One unit of enzyme activity is defined as the amount of enzyme which liberates $1 \mu \mathrm{mol}$ of inorganic phosphorus per min per 10 leaf segments under the assay conditions described.

\section{Results and Discussion}

The changes in the fresh weight and leaf width of leaf segments exposed to the vapor above a $0.5 \mathrm{M} \mathrm{NaCl}$ solution and those floated on distilled water are presented in Fig. 1. In the former leaf segments, fresh weight decreased rapidly for 2 days and leaf width for 1 day, but subsequently remained unchanged. There was a slight but significant increase in the fresh weight of leaf segments floated on water for 3 days. However, no changes of leaf width were found in leaf segments floated on water during a 4-day-incubation.

Recently, O'Toole and Cruz (1980) reported the linear relationship between leaf rolling and leaf water potential and concluded that leaf rolling in rice could be used as an estimate of water deficit. Apparently, leaf rolling occurred in our system 

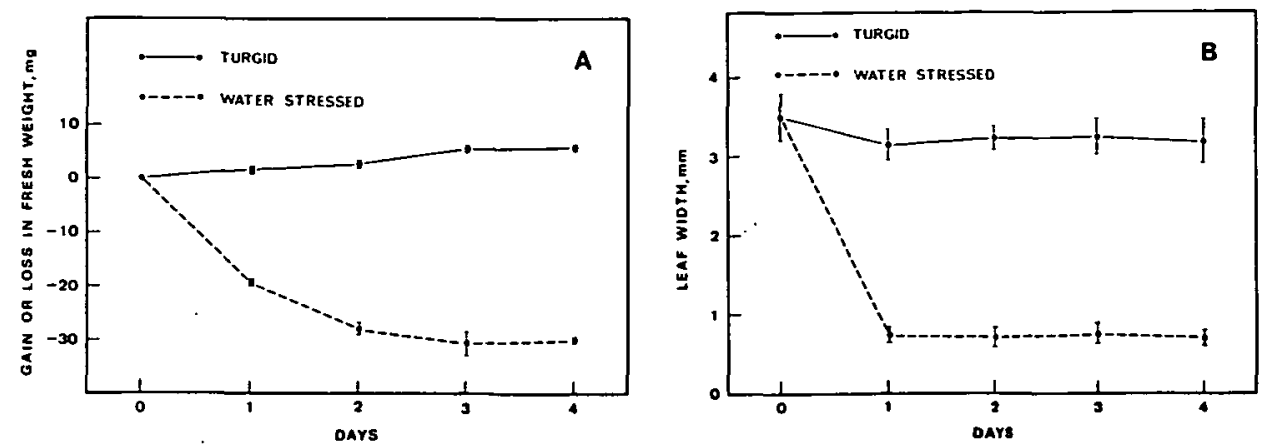

Fig. 1 Changes with time in the fresh weight (A), leaf width (B) and chlorophyll (C) content of detached rice leaves under turgid and waterstressed conditions. Values in $A$ find $B$ are the average with standard errors of 10 leaf segments, while those of $\mathrm{C}$ are the average of triplicate experiments.

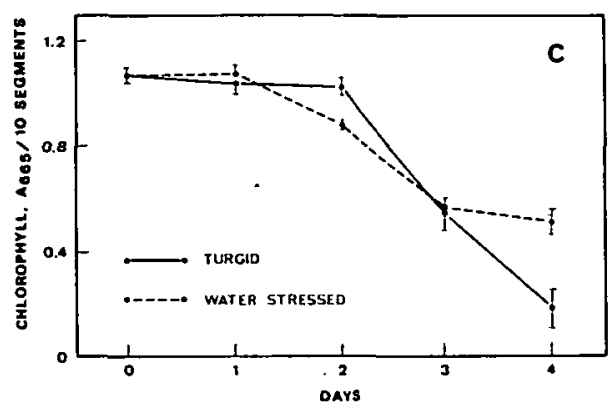

of exposing leaf segments to the atmosphere above a $\mathrm{NaCl}$ solution, thus the treatment indeed caused water stress.

Conventionally, the biochemical parameters used to study the senescence of excised leaves or leaf discs are the decrease in the chlorophyll and protein content and the increase in a-amino nitrogen content (Dwivedi et al. 1979b, Kao 1979, Martin and Thimann 1972, Sugiura et al. 1962). Using these parameters, Dwivedi et al. (1979a) showed that senescence was enhanced by water stress in excised rice leaves. Several investigators also have reported the enhancement of the senescencee of detached and attached leaves and other plant parts by water stress (Brady et al. 1974, Dwivedi et al. 1979b, Gates 1968, Livne and Vaadia 1968, Shah and Loomis 1965).

However, in the present investigation, the decrease in the chlorophyll and protein level and the increase of the $a$-amino nitrogen were accelerated by water stress only during the first two or three days (Fig. 1 and 2); during later incubation, water stress retarded the changes in chlorophyll, protein and $\alpha$-amino nitrogen levels. These results are consistent with our early findings (Kao 1979), that mannitol-stressed intact rice leaves had higher chlorophyll content than turgid intact leaves after four days of dark incubation.

Stewart (1971) reported that the decrease in respiration rate was faster in turgid leaves than in water-stressed leaves. In other words, a faster rate of decline in soluble sugar in the turgid leaves than in the water-stressed leaves is to be expected. Indeed this was the case in this study (Fig. 2). Furthermore, the decrease in starch content has been reported to be slower in turgid leaves than that in stressed leaves 
(Dwivedi et al. 1979a). Therefore, the faster rate of decline in solugle sugar in turgid leaves is indeed due to the higher respiration rate.

An interesting aspect of water stress is the accumulation of the amino acid proline. Several workers (Singh et al. 1973, Stewart et al. 1966, Stewart 1971, Thompson et al. 1966) have reported the striking increase in proline content in stressed plants. But relatively little attention has been paid to the proline accumulation in senescing excised leaves. Thompson et al. (1966) and Stewart et al. (1966) showed that proline did not accumulate in excised, turgid turnip leaves when incubated in the dark. In the present study, however, the proline level increased with time in water-floated turgid leaves, though at a slower rate than the leaves subjected to water stress (Fig. 2). In turgid leaves there was a slight but significant increase in the proline accumulation in the first day of incubation, but, subsequently, proline accumulated relatively rapidly. At 4 days after excision, the proline level in turgid leaves had reached the same level as that in water stressed leaves. The accumulation of proline in turgid leaves is not specific for the rice cultivar used in this study: proline also accumulates in 10 other cultivars of rice leaves during senescence (unpublished data).

The activity of acid inorganic pyrophosphatase was increased but that of alkaline inorganic pyrophosphatase decreased during the senescence of both turgid and water stressed leaves (Fig. 3). Obviously these two activities are due to separate enzyme proteins, which is in agreement with the results of Rauser (1971), Kar and
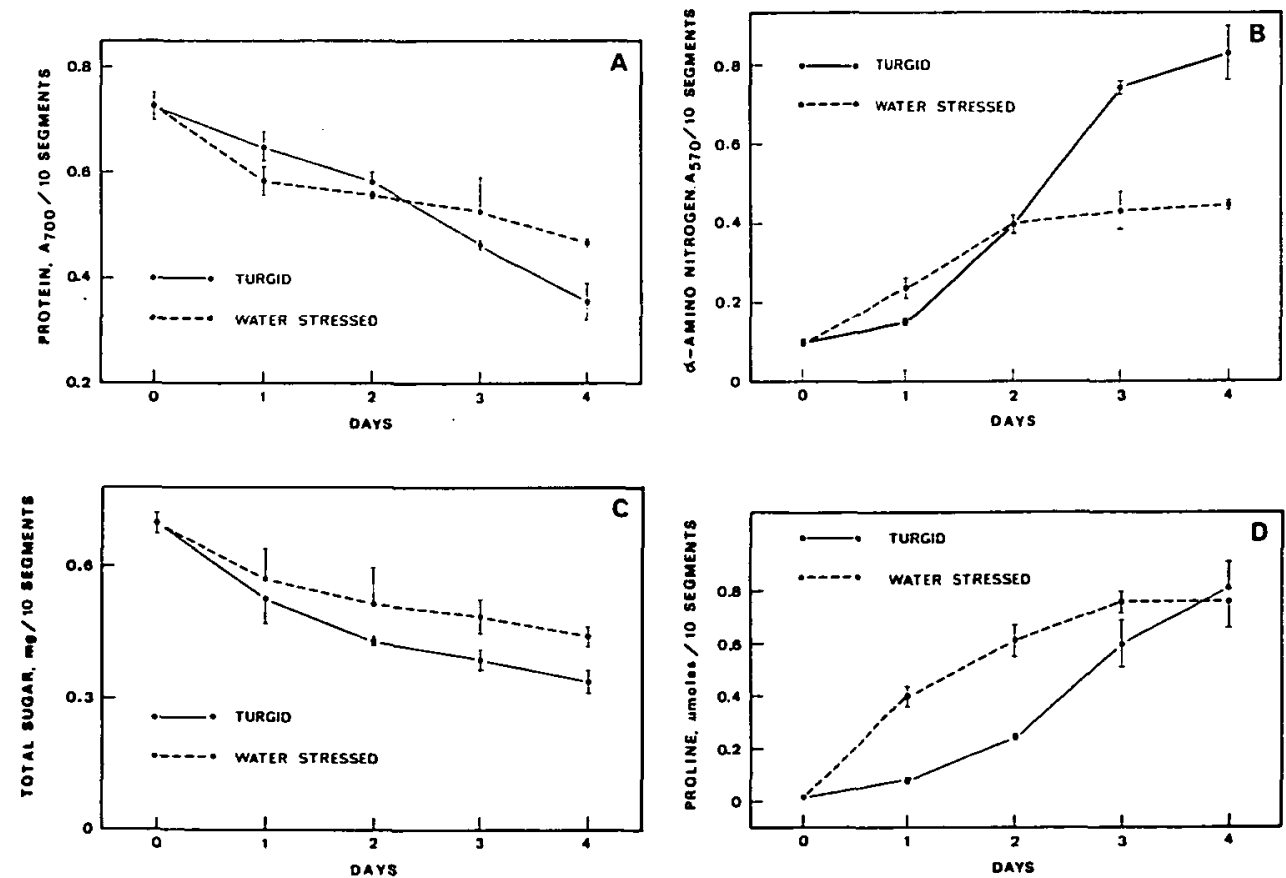

Fig. 2 Changes with time in the protein (A), a-amino nitrogen (B), total soluble sugar (C) and proline content (D) of detached rice leaves under turgid and water-stressed conditions. Values are the average with standard errors of triplicate experiments. 

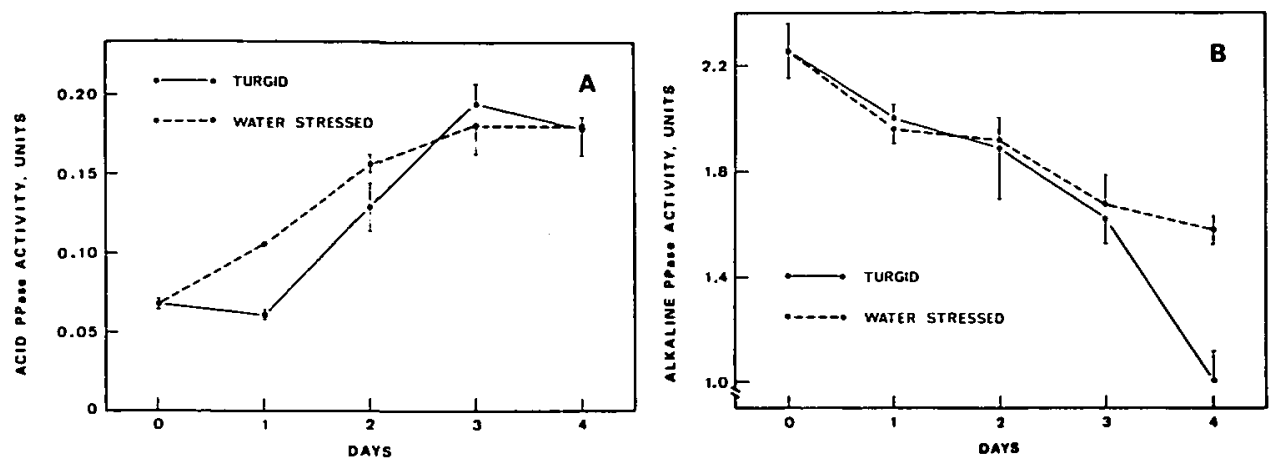

Fig. 3 Changes with time in the inorganic pyrophosphatase (PPase) activities of detached rice leaves under turgid and water-stressed conditions. Values are the average with standard errors of triplicate experiments.

Mishra (1975) and Kar et al. (1978). Several workers (Kar et al. 1978, Rauser 1971, Naganna and Sripathi 1954) reported that acid inorganic pyrophosphatase activity is probably associated with catabolic processes, while alkaline inorganic pyrophosphatase activity is likely associated with anabolic processes. In the present investigation, water stress enhanced the increase of acid inorganic pyrophosphatase activity only during the first two days, but later retarded the decrease of alkaline inorganic pyrophosphatase activity (Fig. 3). It seems that water stressed leaves have a higher rate of catabolic activity in early incubation, and a higher rate of anabolic activity later, compared to turgid leaves. This would explain the observation (Fig. 1 and 2) that the changes in chlorophyll, protein and $\alpha$-amino nitrogen levels were enhanced by water stress during early phase of incubation, but retarded during the later phase.

Although several reports previously cited indicate that water stress enhances senescence, the evidence presented in this paper shows that, under the condition of darkness at least, water stress consistently enhances only some of the metabolic changes associated with the senescence of detached leaves.

The financial support from the National Science Council of the Republic of China is gratefully acknowledged. Mrs. C. P. H. Chang is thanked for her assistance.

\section{References}

Bates, L. S., R. P. Waldren and I. D. Teare (1973) Rapid determination of free proline for water stress studies. Plant and Soil 39: 205-207.

Brady, C. J., N. S. Scott and P. Munns (1974) The interaction of water stress with the senescence pattern of leaves. In Mechanism of Regulation of Plant Growth. Edited by P. L. Bieleski, A. R. Ferguson and M. M. Cresswell. p. 403-409. The Royal Society of New Zealand, Wellington.

Dwivedi, S., M. Kar and D. Mishra (1979a) Biochemical changes in excised leaves of Oryza sativa subjected to water stress. Physiol. Plant. 45: 35-40.

Dwivedi, S., M. Kar and D. Mishra (1979b) Inorganic pyrophosphatase activity in water stressed excised rice leaves. Irrigation Sci. 1: 119-124.

Gates, C. T. (1968) Water deficits and growth of herbaceous plants. In Water Deficits and Plant Growth 2. Edited by T. T. Kozlowski. p. 135-190. Academic Press Ihc., New York, N. Y. 
Kao, C. H. (1979) Senescence of rice leaves III. The inter-organ control of leaf senescence of rice seedlings. Proc. Natl. Sci. Counc. ROC. 3: 199-204.

Kao, C. H. (1980a) Retardation of senescence by low temperature and benzyladenine in intact primary leaves of soybean. Plant \& Cell Physiol. 21 : 339-344.

Kao, C. H. (1980b) Senescence of rice leaves IV. Influence of benzyladenine on chlorophyll degradation. Plant \& Cell Physiol. 21: 1255-1262.

Kar, M. and D. Mishra (1975) Inorganic pyrophosphatase activity during rice leaf senescence. Can. J. Bot. 53: 503-511.

Kar, M., H. K. Patra and D. Mishra (1978) Zn $\mathrm{n}^{2+-D e p e n d e n t ~ a c i d ~ i n o r g a n i c ~ p y r o p h o s p h a t a s e ~}$ activity as related to other pyrophosphatase in Oryza sativa. Physiol. Plant. 43: 287-291.

Livne, A. and Y. Vaadia (1968) Water deficit and hormone relations. In Water Deficits and Plant Growth. Edited by T. T. Kozlowski. p. 255-276. Academic Press Inc., New York, N. Y.

Martin, C. and K. V. Thimann (1972) The role of protein synthesis in the senescence of leaves $I$. The formation of proteases. Plant Physiol. 49: 64-71.

Naganna, B. and C. E. Sripathi (1954) Pyrophosphatase in plants during growth. Nature 174: 593-594.

O'Toole, J. C. and R. T. Cruz (1980) Response of leaf water potential, stomatal resistance, and leaf rolling to water stress. Plant Physiol. 65: 428-432.

Rauser, W. E. (1971) Inorganic pyrophosphatase in leaves during plant development and senescence. Can. J. Bot. 49: 311-316.

Shah, C. B. and R. S. Loomis (1965) Ribonucleic acid and protein metabolism in sugar beet during drought. Physiol. Plant. 18: 240-254.

Singh, T. N., D. Aspinall and I. G. Paleg (1973) Stress metabolism I. Nitrogen metabolism and growth in the barley plant during water stress. Aust. J. Boil. Sci. 26: 45-56.

Stewart, C. R., C. L. Morris and J. F. Thompson (1966) Changes in amino acid content of excised leaves during incubation II. Plant Physiol. 41 : 1585-1590.

Stewart, C. R. (1971) Effect of wilting on carbohydrates during incubation of excised bean leaves in the dark. Plant Physiol. 48: 792-794.

Sugiura, M., K. Umemura and Y. Oota (1962) The effect of kinetin on protein level of tobacco leaf discs. Physiol. Plant. 15: 457-464.

Thompson, J. F., C. R. Stewart and C. J. Morris (1966) Changes in amino acid content of excised leaves during incubation I. Plant Physiol. 41 : 1578-1584.

Yoshida, S., D. A. Forno, J. H. Cock and K. A. Gomez (1972) Laboratory Manual for Physiological Studies of Rice. The International Rice Research Institute, Los Banos, Philippines.

(Received January 24, 1981; Accepted March 27, 1981) 